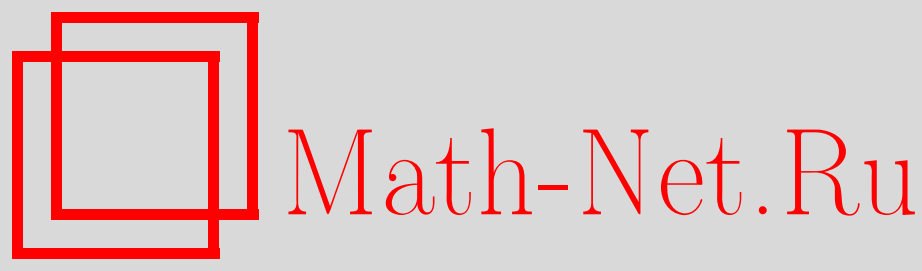

А. А. Славнов, Квантование моделей массивных неабелевых калибровочных полей со спонтанно нарушенной симметрией вне рамок теории возмущений, ТМФ, 2016, том 189, номер 2, 279-285

DOI: https://doi.org/10.4213/tmf9226

Использование Общероссийского математического портала Math-Net.Ru подразумевает, что вы прочитали и согласны с пользовательским соглашением http://www . mathnet.ru/rus/agreement

Параметры загрузки:

IP : 3.80 .253 .173

26 апреля 2023 г., 18:17:17

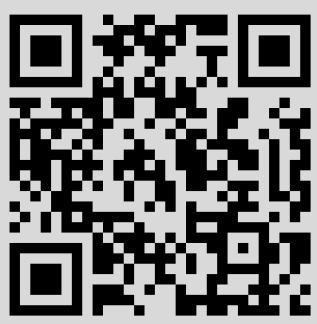




\title{
КВАНТОВАНИЕ МОДЕЛЕЙ МАССИВНЫХ НЕАБЕЛЕВЫХ КАЛИБРОВОЧНЫХ ПОЛЕЙ СО СПОНТАННО НАРУШЕННОЙ СИММЕТРИЕЙ ВНЕ РАМОК ТЕОРИИ ВОЗМУЩЕНИЙ
}

\begin{abstract}
Предложен новый метод однозначного квантования моделей массивных неабелевых калибровочных полей со спонтанно нарушенной симметрией, применимый вне рамок теории возмущений по константе связи.
\end{abstract}

Ключевые слова: модель Англера-Брута-Хиггса-Киббла, перенормировка калибровочно-инвариантных теорий, спонтанное нарушение симметрии.

DOI: $10.4213 / \operatorname{tmf} 9226$

\section{1. ВВЕДЕНИЕ}

Хотя в модели Англера-Брута-Хиггса-Киббла (АБХК) [1]-[3] константа взаимодействия мала, чтобы количественно описать некоторые эффекты, в особенности связанные с открытием бозона Хиггса, необходим выход за рамки теории возмущений. В связи с этим возникает проблема однозначности процедуры квантования. В модели АБХК она имеет специфическую форму. Можно проквантовать теорию в унитарной калибровке, но переход к явно перенормируемой калибровке невозможен вне рамок теории возмущений [4]. Детерминант Фаддеева-Попова, возникающий в процессе такого перехода, становится сингулярным.

Недавно был предложен однозначный метод квантования, допускающий переход к явно перенормируемой калибровке и применимый вне рамок теории возмущений [5]-[9]. Этот метод основан на использовании модифицированного лагранжиана теории Янга-Миллса, который приводит к тем же предсказаниям для наблюдаемых

Исследование выполнено за счет гранта Российского научного фонда (проект № 14-50-00005).

* Математический институт им. В. А. Стеклова РАН, Москва, Россия. E-mail: slavnov@mi.ras.ru 
величин, что и стандартный. Модифицированный лагранжиан содержит дополнительные духовые поля, но также обладает дополнительной (супер)симметрией. Особую актуальность этот метод приобретает при рассмотрении топологически нетривиальных конфигураций, которые существенны для выхода за рамки теории возмущений. Другие подходы к этой проблеме можно найти в статье [10].

В работе [11] данный метод был применен к модели АБХК. В настоящей работе мы также применяем этот метод к модели АБХК, но используем другой способ, который ближе к оригинальной процедуре Фаддеева-Попова и, по нашему мнению, проще, чем метод из работы [11].

\section{2. ОПИСАНИЕ МОДЕЛИ}

Напомним основные идеи метода модифицированных лагранжианов, используя в качестве примера квантовую электродинамику (КЭД). Построение явно лоренцинвариантной локальной формулировки КЭД невозможно без введения вектор-потенциала $A_{\mu}$, который описывает не только физические возбуждения, отвечающие трехмерно поперечным компонентам, но также продольные и временные фотоны. При этом временные фотоны имеют отрицательную энергию. В то же время действие, записанное в терминах вектор-потенциала, обладает дополнительной симметрией. Оно является калибровочно-инвариантным. Такая ситуация типична для теории поля - вводятся нефизические степени свободы и одновременно вводится новая симметрия, которая обеспечивает их отщепление от физических степеней свободы. Дополнительная симметрия, которой обладает теория в такой формулировке, может быть полезна для изучения различных аспектов теории, которые не очевидны в стандартной формулировке. Такой подход используется, например, при БРСТквантовании калибровочно-инвариантных систем.

Имея это в виду, мы предлагаем для описания модели АБХК лагранжиан, который содержит дополнительные духовые поля:

$$
\begin{aligned}
L= & -\frac{1}{4} F_{\mu \nu}^{a} F_{\mu \nu}^{a}+\left(D_{\mu} \varphi_{+}\right)^{*}\left(D_{\mu} \varphi_{-}\right)+\left(D_{\mu} \varphi_{-}\right)^{*}\left(D_{\mu} \varphi_{+}\right)- \\
& -\left[\left(D_{\mu} \varphi_{+}\right)^{*}\left(D_{\mu} \hat{m}\right)+\left(D_{\mu} \hat{m}\right)^{*}\left(D_{\mu} \varphi_{+}\right)\right]-\left[\left(D_{\mu} b\right)^{*}\left(D_{\mu} e\right)+\left(D_{\mu} e\right)^{*}\left(D_{\mu} b\right)\right]+ \\
& +\left(D_{\mu} \varphi\right)^{*}\left(D_{\mu} \varphi\right)-\left[\left(D_{\mu} \varphi\right)^{*}\left(D_{\mu} \hat{m}\right)+\left(D_{\mu} \hat{m}\right)^{*}\left(D_{\mu} \varphi\right)\right]+ \\
& +\left(D_{\mu} \hat{m}\right)^{*}\left(D_{\mu} \hat{m}\right)-\lambda^{2}\left[(\varphi-\hat{m})^{*}(\varphi-\hat{m})-m^{2}\right]^{2} .
\end{aligned}
$$

Строго говоря, этот лагранжиан описывает массивные калибровочные поля, взаимодействующие с одним скалярным полем. Однако, поскольку в нем присутствуют основные трудности, связанные с введением массивных калибровочных полей, мы в целях экономии места будем называть его лагранжианом модели АБХК. Лагранжиан (1) получается путем сдвига полей в калибровочно-инвариантном лагранжиане

$$
\begin{aligned}
L= & -\frac{1}{4} F_{\mu \nu}^{a} F_{\mu \nu}^{a}+\left(D_{\mu} \varphi_{+}\right)^{*}\left(D_{\mu} \varphi_{-}\right)+\left(D_{\mu} \varphi_{-}\right)^{*}\left(D_{\mu} \varphi_{+}\right)- \\
& -\left[\left(D_{\mu} b\right)^{*}\left(D_{\mu} e\right)+\left(D_{\mu} e\right)^{*}\left(D_{\mu} b\right)\right]+\left(D_{\mu} \varphi\right)^{*}\left(D_{\mu} \varphi\right)-\lambda^{2}\left[\varphi^{*} \varphi-m^{2}\right]^{2},
\end{aligned}
$$


поэтому он, очевидно, инвариантен относительно "сдвинутых" калибровочных преобразований. Явный вид преобразования, результатом которого является лагранжиан (1), следующий:

$$
\varphi_{-}(x) \rightarrow \varphi_{-}(x)-\hat{m}, \quad \varphi(x) \rightarrow \varphi(x)-\hat{m} .
$$

В этих формулах $\hat{m}=(0, m)$ - двухкомпонентная величина, компонентами которой являются константы.

Закон преобразования "сдвинутых" переменных выглядит следующим образом:

$$
\begin{array}{rlrl}
\delta A_{\mu}^{a}=\partial_{\mu} \eta^{a}+g \epsilon^{a b c} A_{\mu}^{b} \eta^{c}, & \\
\delta \varphi_{+}^{a} & =\frac{g}{2} \epsilon^{a b c} \varphi_{+}^{b} \eta^{c}+\frac{g}{2} \varphi_{+}^{0} \eta^{a}, & \delta \varphi_{+}^{0} & =-\frac{g}{2} \varphi_{+}^{a} \eta^{a}, \\
\delta \varphi_{-}^{a} & =m \eta^{a}+\frac{g}{2} \epsilon^{a b c} \varphi_{-}^{b} \eta^{c}+\frac{g}{2} \varphi_{-}^{0} \eta^{a}, & \delta \varphi_{-}^{0} & =-\frac{g}{2} \varphi_{-}^{a} \eta^{a}, \\
\delta \varphi^{a} & =m \eta^{a}+\frac{g}{2} \epsilon^{a b c} \varphi^{b} \eta^{c}+\frac{g}{2} \varphi^{0} \eta^{a}, & \delta \varphi^{0} & =-\frac{g}{2} \varphi^{a} \eta^{a}, \\
\delta b^{a} & =\frac{g}{2} \epsilon^{a b c} b^{b} \eta^{c}+\frac{g}{2} b^{0} \eta^{a}, & \delta b^{0} & =-\frac{g}{2} b^{a} \eta^{a}, \\
\delta e^{a} & =\frac{g}{2} \epsilon^{a b c} e^{b} \eta^{c}+\frac{g}{2} e^{0} \eta^{a}, & \delta e^{0} & =-\frac{g}{2} e^{a} \eta^{a}
\end{array}
$$

и

$$
\Phi=\left(\frac{i \Phi_{1}+\Phi_{2}}{\sqrt{2}} ; \frac{\Phi_{0}-i \Phi_{3}}{\sqrt{2}}\right) .
$$

Все поля, входящие в преобразования (4), имеют вид $(5)$, где $\Phi_{i}-$ эрмитовы компоненты. Поля $b, e$ параметризованы аналогичным образом, но в отличие от полей $\varphi$, $\varphi_{ \pm}$, эти поля антикоммутируют. По определению поле $e$ параметризовано эрмитовыми компонентами, а компоненты поля $b$ антиэрмитовы.

Как видно, обе переменные $\varphi^{a}$ и $\varphi_{-}^{a}$ при калибровочном преобразовании сдвигаются на произвольную функцию $\eta^{a}$, т. е. играют роль калибровочных полей. Чтобы проквантовать теорию, нужно наложить калибровочное условие на $A_{\mu}$ или одну из этих функций. Наложение калибровочного условия на $A_{\mu}$ приводит к неоднозначности Грибова. Следовательно, мы должны наложить калибровочное условие на $\varphi^{a}$ или $\varphi_{-}^{a}$. Мы выберем “унитарную” калибровку $\varphi^{a}=0$. В этой калибровке спектр теории включает кроме стандартного массивного векторного поля и бозона Хиггса также поля $b, e, \varphi_{ \pm}$. Поля $\varphi_{ \pm}$и $b, e$ компенсируют друг друга. В самом деле, если поля $\varphi_{ \pm}, b, e$ отсутствуют в асимптотических состояниях, то в континуальном интеграле, описывающем матрицу рассеяния для лагранжиана (2), можно проинтегрировать по полям $\varphi_{ \pm}, b, e$, и возникающие при этом детерминанты будут компенсировать друг друга.

В общем случае духовые поля $\varphi_{ \pm}, b, e$ присутствуют в асимптотических состояниях, однако лагранжианы (1), (2) обладают дополнительной инвариантностью по сравнению со стандартным лагранжианом модели АВХК. Эти лагранжианы инвариантны относительно преобразований суперсимметрии

$$
\delta \varphi_{-}(x)=b(x) \epsilon, \quad \delta e(x)=\varphi_{+}(x) \epsilon, \quad \delta b(x)=i \delta \varphi_{+}=0 .
$$

В силу теоремы Нётер инвариантность лагранжиана (1) относительно преобразований (6) порождает сохраняющийся заряд $Q_{\mathrm{S}}$. Согласно постановке задачи рассеяния 
в квантовой теории взаимодействие между полями обращается в нуль при $t \rightarrow \pm \infty$, и поля становятся невзаимодействующими. Поэтому мы можем наложить на асимптотические состояния условие

$$
Q_{\mathrm{S}}^{\mathrm{as}}|\psi\rangle^{\mathrm{as}}=0,
$$

где асимптотические поля удовлетворяют свободным полевым уравнениям и, следовательно, асимптотический заряд есть асимптотическое значение оператора $Q_{\mathrm{S}}$. Аналогичное условие можно наложить на асимптотические состояния в силу сохранения БРСТ-заряда $Q_{\mathrm{B}}$, однако для случая “унитарной” калибровки оно, как мы покажем, будет тривиальным:

$$
Q_{\mathrm{B}}^{\mathrm{as}}|\psi\rangle^{\mathrm{as}}=0
$$

Как легко видеть,

$$
\left[Q_{\mathrm{B}}^{\mathrm{as}}, Q_{\mathrm{S}}^{\mathrm{as}}\right]=0
$$

поэтому условия (7) и (8) совместны.

"Унитарная" калибровка $\varphi^{a}=0$ является алгебраической, соответствующие духи Фаддеева-Попова ультралокальны и не дают вклада в наблюдаемые величины. Для дальнейшего анализа удобно ввести новые переменные $\tilde{\varphi}_{ \pm}, \tilde{b}, \tilde{e}$ с помощью равенств

$$
\begin{gathered}
\varphi_{+}^{a}=M^{-1} \tilde{\varphi}_{+}^{a}, \quad \varphi_{+}^{0}=M^{-1} \tilde{\varphi}_{+}^{0}, \quad \varphi_{-}^{a}=M \tilde{\varphi}_{-}^{a}, \quad \varphi_{-}^{0}=\frac{2 m}{g}\left(e^{g h / 2 m}-1\right), \\
b=M \tilde{b}, \quad e=M^{-1} \tilde{e},
\end{gathered}
$$

где

$$
M=1+\frac{g}{2 m} \varphi_{-}^{0}=e^{g h / 2 m} .
$$

Преобразованный таким образом лагранжиан, выраженный через переменные $h$, $\tilde{\varphi}_{ \pm}, \tilde{b}, \tilde{e}$, по-прежнему инвариантен относительно суперпреобразований и БРСТпреобразований. В частности, преобразования суперсимметрии в новых переменных имеют вид

$$
\begin{array}{rlrl}
\delta \tilde{\varphi}_{+}^{a} & =-\frac{g}{2 m} \tilde{b}^{0} \tilde{\varphi}_{+}^{a}, & \delta \tilde{\varphi}_{+}^{0} & =-\frac{g}{2 m} \tilde{b}^{0} \tilde{\varphi}_{+}^{0}, \\
\delta \tilde{\varphi}_{-}^{a} & =-\tilde{b}^{a}+\frac{g}{2 m} \tilde{\varphi}_{-}^{a} \tilde{b}^{0}, & & \\
\delta \tilde{e}^{a} & =\tilde{\varphi}_{+}^{a}+\frac{g}{2 m} \tilde{e}^{a} \tilde{b}^{0}, & \delta \tilde{e}^{0} & =\tilde{\varphi}_{+}^{0}+\frac{g}{2 m} \tilde{e}^{0} \tilde{b}^{0}, \\
\delta \tilde{b}^{a} & =-\frac{g}{2 m} \tilde{b}^{a} \tilde{b}^{0}, & \delta \tilde{b}^{0} & =0, \\
\delta h=-\tilde{b}^{0} . &
\end{array}
$$

Квантование в калибровке $\varphi^{a}=0$ производится стандартным образом. В силу того что мы рассматриваем калибровку $\varphi^{a}=0$, которая является алгебраической, все возникающие при квантовании духовые поля ультралокальны и не дают вклада в наблюдаемые величины. Спектр теории состоит из массивного векторного поля, скалярного поля Хиггса и духовых полей $\varphi_{ \pm}, b, e$, которые, как мы покажем, отщепляются от физических состояний. Заметим, что $Q_{\mathrm{S}}^{\mathrm{as}}=Q_{\mathrm{S}}^{0}$ и $Q_{\mathrm{B}}^{\mathrm{as}}=Q_{\mathrm{B}}^{0}$, где $Q_{\mathrm{S}}^{0}=Q_{\mathrm{S}}$ и $Q_{\mathrm{B}}^{0}=Q_{\mathrm{B}}$ при $g=0$. Поэтому мы можем считать, что условие $(9)$ выполнено. 
Оператор $Q_{\mathrm{S}}^{\mathrm{as}}$ имеет вид

$$
\begin{aligned}
Q_{\mathrm{S}}^{\mathrm{as}}=\int d^{3} x\left[\partial_{0}\left(\tilde{\varphi}_{+}^{\mathrm{as}}\right)^{*}(x) \tilde{b}^{\mathrm{as}}(x)+\partial_{0}\left(\tilde{\varphi}_{+}^{\mathrm{as}}\right)(x)\left(\tilde{b}^{\mathrm{as}}\right)^{*}(x)-\right. \\
\left.-\partial_{0}\left(\tilde{b}^{\mathrm{as}}\right)^{*}(x) \tilde{\varphi}_{+}^{\mathrm{as}}(x)-\partial_{0} \tilde{b}^{\mathrm{as}}(x)\left(\tilde{\varphi}_{+}^{\mathrm{as}}\right)^{*}(x)\right] .
\end{aligned}
$$

Этот заряд, очевидно, асимптотически сохраняется, так как асимптотические поля удовлетворяют уравнениям

$$
\partial_{x}^{2} \tilde{\varphi}_{+}^{\mathrm{as}}(x)=\partial_{x}^{2} \tilde{b}^{\mathrm{as}}(x)=0
$$

Можно ввести оператор

$$
\begin{array}{r}
K_{\mathrm{S}}=\int d^{3} x\left[\left(\tilde{\varphi}_{-}^{\mathrm{as}}\right)^{*}(x) \partial_{0} \tilde{e}^{\mathrm{as}}(x)+\tilde{\varphi}_{-}^{\mathrm{as}}(x) \partial_{0}\left(\tilde{e}^{\mathrm{as}}\right)^{*}(x)-\right. \\
\left.-\left(\tilde{e}^{\mathrm{as}}\right)^{*}(x) \partial_{0} \tilde{\varphi}_{-}^{\mathrm{as}}(x)-\tilde{e}^{\mathrm{as}}(x) \partial_{0}\left(\tilde{\varphi}_{-}^{\mathrm{as}}\right)^{*}(x)\right]
\end{array}
$$

такой, что

$$
\left[K_{\mathrm{S}}, Q_{\mathrm{S}}^{\mathrm{as}}\right]_{+} \sim R
$$

где $R$ - оператор числа нефизических частиц $\tilde{\varphi}_{ \pm}, \tilde{b}, \tilde{e}$. Следовательно, любое состояние, анигилируемое зарядом $Q_{\mathrm{S}}^{\mathrm{as}}$, имеет структуру

$$
|\psi\rangle^{\text {as }}=|\psi\rangle_{0}+|N\rangle
$$

где состояние $\left|\psi_{0}\right\rangle$ не содержит духовых полей $\tilde{\varphi}_{ \pm}, \tilde{b}, \tilde{e}$, а состояние $|N\rangle$ описывает вектор с нулевой нормой, ортогональный любому состоянию, которое не содержит духовых полей $\tilde{\varphi}_{ \pm}, \tilde{b}, \tilde{e}$.

Если принять во внимание, что лагранжиан (1) в калибровке $\varphi^{a}=0$ БРСТ-инвариантен, а члены, порождаемые ультралокальными духами Фаддеева-Попова, не дают вклада в наблюдаемые величины, легко показать, что наша теория эквивалентна стандартной. Чтобы обеспечить полную эквивалентность, нужно факторизовать пространство состояний, удовлетворяющих условиям (7), (8), по векторам с нулевой нормой.

Итак мы доказали, что квантование лагранжиана (1) приводит к теории, эквивалентной стандартной модели АБХК в унитарной калибровке.

Калибровка $\varphi^{a}=0$ не является явно перенормируемой. Однако калибровочная инвариантность теории позволяет легко перейти к явно перенормируемой калибровке, применимой вне рамок теории возмущений. Это можно сделать при помощи трюка Фаддеева-Попова. Умножим матрицу рассеяния в калибровке $\varphi^{a}=0$ на "единицу", записанную в форме

$$
\Delta \int d \Omega \delta\left(\left[\varphi_{-}^{\Omega}\right]^{a}\right)=1
$$

где интегрирование ведется по инвариантной мере на калибровочной группе. Функционал $\Delta$, очевидно, калибровочно инвариантен и не дает вклада в наблюдаемые величины. Поэтому в матрице рассеяния, умноженной на выражение (18), можно сделать замену переменных, которая является калибровочным преобразованием 
$\left[\tilde{\varphi}_{-}^{\Omega}\right]^{a}=\tilde{\varphi}_{-}^{a}$. Полученный эффективный лагранжиан (если опустить члены, отвечающие вкладу локальных духов, которые не дают вклада в наблюдаемые величины) совпадает с лагранжианом, приведенным в работе [11] для калибровки $\varphi_{-}^{a}=0$. Он выглядит следующим образом:

$$
\begin{aligned}
\tilde{L}= & -\frac{1}{4} F_{\mu \nu}^{a} F_{\mu \nu}^{a}+\partial_{\mu} h \partial_{\mu} \tilde{\varphi}_{+}^{0}-\frac{g}{2 m} \partial_{\mu} h \partial_{\mu} h \tilde{\varphi}_{+}^{0}+ \\
& +m \tilde{\varphi}_{+}^{a} \partial_{\mu} A_{\mu}^{a}-\left[\left(D_{\mu} b\right)^{*}+\frac{g}{2 m} \tilde{b}^{*} \partial_{\mu} h\left(D_{\mu} e-\frac{g}{2 m} \tilde{e} \partial_{\mu} h\right)\right]+ \\
& +\left[\left(D_{\mu} b\right)+\frac{g}{2 m} \tilde{b} \partial_{\mu} h\left(D_{\mu} e^{*}-\frac{g}{2 m} \tilde{e}^{*} \partial_{\mu} h\right)\right]+ \\
& +\frac{m g}{2} A_{\mu}^{2} \tilde{\varphi}_{+}^{0}+g \partial_{\mu} h A_{\mu}^{a} \tilde{\varphi}_{+}^{a}+\frac{m^{2}}{2} A_{\mu}^{a} A_{\mu}^{a}+m \varphi^{a} \partial_{\mu} A_{\mu}^{a}+\cdots
\end{aligned}
$$

Из членов, возникающих в модели АБХК, мы выписали явно только массовый член и квадратичный член, описывающий смешивание голдстоуновского бозона с калибровочным, остальные члены обозначены многоточием. Перекрестный член можно устранить, сделав замену переменных

$$
\tilde{\varphi}_{+}^{a} \rightarrow \tilde{\varphi}_{+}^{a}-\varphi^{a}
$$

Свободные пропагаторы имеют вид

$$
\begin{gathered}
D\left(A_{\mu}^{a} A_{\nu}^{b}\right)=-\frac{\delta^{a b}}{p^{2}-m^{2}} T_{\mu \nu}, \quad D\left(A_{\mu}^{a} \tilde{\varphi}_{+}^{b}\right)=-\delta^{a b} \frac{p_{\mu}}{m p^{2}}, \quad D\left(\tilde{\varphi}_{+}^{0} h\right)=\frac{i}{p^{2}}, \\
D\left(\tilde{b}^{0} \tilde{e}^{0}\right)=\frac{i}{p^{2}}, \quad D\left(\tilde{b}^{a} \tilde{e}^{b}\right)=\frac{i \delta^{a b}}{p^{2}} \\
D\left(\varphi^{0} \varphi^{0}\right)=\frac{i}{p^{2}-M_{\mathrm{H}}^{2}}, \quad D\left(\varphi^{a} \varphi^{b}\right)=\frac{i \delta^{a b}}{p^{2}},
\end{gathered}
$$

где $T_{\mu \nu}$ - поперечный проектор, $M_{\mathrm{H}}^{2}=4 m^{2} \lambda^{2}$ - масса мезона Хиггса.

Индекс расходимости произвольной диаграммы равен

$$
n=4-2 L_{\tilde{\varphi}_{+}^{a}}-2 L_{\tilde{\varphi}_{+}^{0}}-L_{A}-L_{e}-L_{b}-L_{h}-L_{\varphi^{a}}-L_{\varphi^{0}} .
$$

Расходящиеся диаграммы могут иметь не больше четырех внешних линий. Теория явно перенормируема.

\section{3. ЗАКЛЮЧЕНИЕ}

В отличие от работы [11], мы проквантовали теорию не в явно перенормируемой калибровке $\tilde{\varphi}_{-}^{a}=0$, а в калибровке $\varphi^{a}=0$, в которой без труда можно доказать унитарность. Затем с помощью преобразования, не меняющего матрицу рассеяния, мы показали, что на массовой поверхности она совпадает с явно перенормируемой матрицей рассеяния в калибровке $\tilde{\varphi}_{-}^{a}=0$. Таким образом, матрица рассеяния обладает как унитарностью, так и перенормируемостью. Строго говоря, следует также доказать, что процедура перенормировки совместна с калибровочной инвариантностью. Это можно сделать с помощью инвариантной регуляризации или следуя работе [11]. 


\section{Список литературы}

[1] F. Englert, R. Brout, Phys. Lett., 13:9 (1964), 321-323.

[2] P. W. Higgs, Phys. Lett., 12:2 (1964), 132-133; Phys. Rev., 145:4 (1966), 1156-1163.

[3] T. W. B. Kibble, Phys.Rev., 155:5 (1967), 1554-1561.

[4] V. N. Gribov, Nucl. Phys. B, 139:1-2 (1978), 1-19.

[5] A. A. Slavnov, JHEP, 08 (2008), 047, 11 pp., arXiv: 0807.1795.

[6] А. А. Славнов, ТМФ, 161:2 (2009), 204-211.

[7] A. Quadri, A. A. Slavnov, JHEP, 07 (2010), 087, 22 pp., arXiv: 1002.2490.

[8] А. А. Славнов, Тр. МИАН, 272 (2011), 246-255.

[9] А.А. Славнов, ТМФ, 183:2 (2015), 163-176.

[10] Д. В. Быков, ТМФ, 185:2 (2015), 313-328.

[11] А. Квадри, А. А. Славнов, ТМФ, 166:3 (2011), 336-349. 